\title{
RADIATION PHENOMENA AT EDGE AND DIVERTOR
}

\author{
U. Samm \\ Institut für Plasmaphysik \\ Forschungszentrum Jülich, Association EURATOM-KFA, Jülich, Germany, Member in the Trilateral Euregio Cluster
}

\begin{abstract}
The concept of a cold radiative plasma boundary is presented as a possible solution of the energy exhaust problem in a fusion reactor. The most relevant processes which determine level and location of the radiation from low- $Z$ impurities are discussed in detail. Experimental results are used to demonstrate the general feasibility of generating a stable and quasi-stationary plasma with a cold radiating layer on a high power level. Furthermore, the limitations of the concept are briefly analyzed addressing feed-back control of the impurity level, thermal stability, particle transport and impurity contamination.
\end{abstract}

\section{THE CONCEPT OF THE "COLD RADIATIVE PLASMA BOUNDARY"}

One of the outstanding challenges on the way to a fusion reactor is the problem of energy exhaust ${ }^{1}$. The $\alpha$-particle heating will generate a radial power flow of about $0.3 \mathrm{MW} / \mathrm{m}^{2}$ which ultimately has to be exhausted via the wall structures (ITER: $\mathrm{P}_{\alpha} \approx 100 \mathrm{MW}$ ). If most of this power would enter the scrape-off-layer (SOL) convectively the power flowing parallel to the fieldlines would be in the order of 500 $\mathrm{MW} / \mathrm{m}^{2}$. Even with optimistic assumptions about possible means for reducing the power load density on the wall surface, e.g. by an inclined target plate, the peak load on the first wall will come close to $20 \mathrm{MW} / \mathrm{m}^{2}$ - a value too high for stationary operation. The necessary reduction of the power load can be achieved by distributing a substantial fraction of the power on other parts of the wall. A promising means to achieve this is given by line radiation from impurities, which allows to distribute the power load on large areas (the whole vessel/divertor wall). In case this line radiation would be confined to the plasma boundary, cooling the plasma edge and leaving the plasma core essentially unaffected, we have a situation as considered in the concept of the "cold radiative plasma boundary".

\section{I.A. The relevant processes and the radiation characteristics}

Impurities enter the plasma in general as neutral particles - as atoms or molecules. Due to electron impact the particles are dissociated and ionized. The characteristic penetration depth $\lambda_{\mathrm{i}}$ of the particles is given by their ionization time

$$
\tau_{\mathrm{i}}=1 /\left(\mathrm{n}_{\mathrm{e}}<\sigma_{\mathrm{i}} \mathrm{v}>\right)
$$

and the velocity of the atoms $\mathrm{v}_{\mathrm{A}}$ according to

$$
\lambda_{\mathrm{i}}=\mathrm{v}_{\mathrm{A}} \tau_{\mathrm{i}}
$$

where $n_{e}$ is the local electron density and $\left\langle\sigma_{\mathrm{i}} \mathrm{v}\right\rangle$ is the corresponding rate coefficient for ionization. Typical values of $\lambda_{i}$ for neutrals are in the order of $1 \mathrm{~cm}$; e.g. for neon injected via a gas puff system or for sputtered silicon. After ionization the charged particles are transported in radial direction by cross-field diffusion. The characteristic transport length is given again by equ. 2 , but with $\mathrm{v}_{\mathrm{A}}$ replaced but an effective radial diffusion velocity $\mathrm{v} \perp$. Step by step ionization to higher states occurs as the particles penetrate into regions with higher electron temperatures $\mathrm{T}_{\mathrm{e}}$. Recombination does not play a role at the plasma edge since the typical times for recombination at densities in the range of $n_{e}=10^{13} \mathrm{~cm}^{-3}$ are for most cases orders of magnitude longer than the average dwell time of the particles in the plasma. In contrast, inside a divertor with densities sometimes above $10^{14} \mathrm{~cm}^{-3}$ recombination may become relevant. However, in an edge plasma the distribution of the ion charge states forms radial nested regions with increasing charge towards the plasma centre.

Excitation of the particles is closely linked to the ionisation process. The excitation rate is given by

$$
\varphi_{\mathrm{z}}{ }^{\mathrm{ij}}=\mathrm{n}_{\mathrm{e}} \mathrm{n}_{\mathrm{iz}}<\sigma_{\mathrm{e}} \mathrm{v}>_{\mathrm{z}}{ }^{\mathrm{ij}},
$$

where $\mathrm{n}_{\mathrm{iz}}$ is the local ion density of a given charge state $\mathrm{z}$ and $\left.<\sigma_{\mathrm{e}} \mathrm{v}\right\rangle_{\mathrm{z}}{ }_{\mathrm{i}}{ }^{\mathrm{ij}}$ is the rate coefficient for the transition $\mathrm{i}-\mathrm{j}$. Excitation can only take place during the life time $\tau_{\mathrm{i}}$ of the ion, thus the total number of excitations is $\mathrm{N}_{\mathrm{z}}{ }^{\mathrm{ij}}=\varphi_{\mathrm{z}}{ }^{\mathrm{ij}} \tau_{\mathrm{i}}$ or the number of excitations per ion is given by the ratio of the rate coefficients

$$
\alpha_{\mathrm{z}}^{\mathrm{ij}}=<\sigma_{\mathrm{e}} \mathrm{v}>_{\mathrm{z}}^{\mathrm{ij}} /<\sigma_{\mathrm{i}} \mathrm{v}>
$$




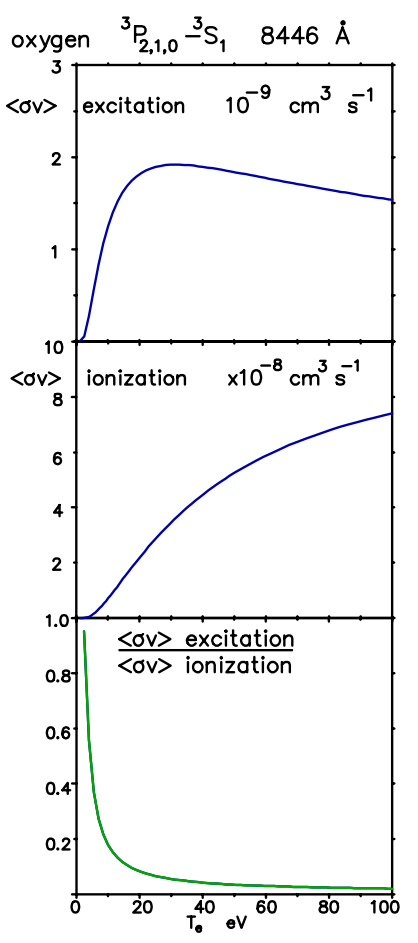

Figure 1 Example for the $T_{e}$-dependence of rate coefficients and their ratio

The rate coefficients depend mainly on $\mathrm{T}_{\mathrm{e}}$. A typical example is shown in Fig. 1 for a certain transition with oxygen ${ }^{2,3}$. By multiplying $\alpha_{\mathrm{z}}^{\mathrm{ij}}$ with the excitation energy $\Delta \mathrm{E}^{\mathrm{j}}$ one obtains the power which can be radiated from this state. Summation over all energy levels and charge states with relative concentrations $c_{z}$ of the impurity particle gives the total energy radiated during its life time in the plasma.

$$
\mathrm{E}_{\mathrm{rad}}=\sum_{\mathrm{z}} c_{z} \sum_{\mathrm{ij}}\left(\alpha_{\mathrm{z}}^{\mathrm{ij}} \Delta \mathrm{E}^{\mathrm{j}}\right)
$$

The term $\mathrm{E}_{\mathrm{rad}}$ is called the radiation potential. The total power radiated $\mathrm{P}_{\text {rad }}$ is then obtained by multiplying $\mathrm{E}_{\mathrm{rad}}$ with the total flux $\Gamma_{\mathrm{i}}$ of the impurity particles entering the plasma (usually as neutrals)

$$
\mathrm{P}_{\mathrm{rad}}=\mathrm{E}_{\mathrm{rad}} \Gamma_{\mathrm{i}}
$$

Radial transport and the $T_{e}$ profiles determine the relative population of the various ionization stages. The ionization time $\tau_{\mathrm{i}}$ for certain ionization states depends on the local $T_{e}$ values. However, radial transport limits essentially the overall life time (dwell time) of the specific ion in the plasma: either by neutralization at the wall or by ionization to higher states.

If transport is diminishing $(\mathrm{v} \perp \rightarrow 0)$ we have the special case of coronal equilibrium, where the dwell time approaches infinity and radiative recombination becomes relevant. In this case the radiation potential has no meaning anymore. Instead, the radiation function $\mathrm{P}\left(\mathrm{T}_{\mathrm{e}}\right)$ can be used to calculate the local radiation density $\mathrm{p}_{\mathrm{rad}}$ :

$$
\mathrm{p}_{\text {rad }}=\mathrm{P}\left(\mathrm{T}_{\mathrm{e}}\right) \mathrm{n}_{\mathrm{e}} \mathrm{n}_{\mathrm{i}} .
$$

Here $n_{i}$ is the ion density summed over all ionization states. The total power radiated is obtained by integrating over the whole plasma volume

$$
\mathrm{P}_{\mathrm{rad}}=\iiint \mathrm{n}_{\mathrm{e}} \mathrm{n}_{\mathrm{i}} \mathrm{P}\left(\mathrm{T}_{\mathrm{e}}\right) \mathrm{dx} \mathrm{dy} \mathrm{dz} .
$$

An example for $\mathrm{P}\left(\mathrm{T}_{\mathrm{e}}\right)$ of some elements ${ }^{4,5}$ is shown in Fig. 2.
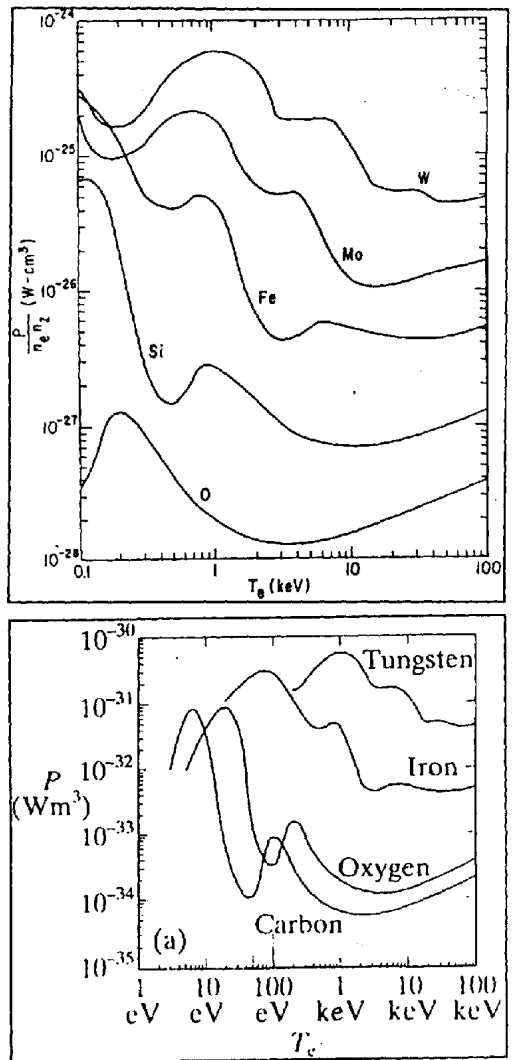

Figure 2 Radiation function for coronal equilibrium from Jensen, Post $^{4}$ and Summers, McWhirter ${ }^{5}$

The advantage of relation 6 is that $\Gamma_{\mathrm{i}}$ can be measured much easier than the radial distribution of ion densities as required in equ. 8 . The radiation potential helps to identify the impurity flux needed for a certain power level of radiation and to qualify the capability for radiation cooling of different impurities, whereas equ. 8 relates radiation to the impurity density at the boundary. Both terms, $\mathrm{E}_{\mathrm{rad}}$ and $\mathrm{P}\left(\mathrm{T}_{\mathrm{e}}\right)$, depend on transport properties of the impurity particles and are most sensitive to the local electron temperature.

But coronal equilibrium is not valid at the plasma boundary since it requires a balance between ionization and radiative recombination. Instead, the ionization equilibrium is determined by transport. In 0 $\mathrm{d}$ calculations $\left(\mathrm{v}=0\right.$, constant $\left.\mathrm{T}_{\mathrm{e}}\right)$ the ionization equilibrium may be 
approximated by introducing a finite lifetime ${ }^{6}$. But a more accurate calculation requires at least a 1-d treatment of the transport processes. However, both methods show that the existence of lower ionization states can be shifted significantly towards higher temperatures yielding much more radiation at higher $\mathrm{T}_{\mathrm{e}}$ compared to the coronal equilibrium case. For example the peak radiation of carbon shown in Fig. 3, calculated with a 1-d transport model ${ }^{7}$, occurs at $\mathrm{T}_{\mathrm{e}}=35 \mathrm{eV}$, a value much higher than the peak appearing at $\mathrm{T}_{\mathrm{e}}=7-8 \mathrm{eV}$ in Fig. 2 . We conclude that the transport dominated ionization equilibrium is advantageous for providing a high radiation level for low- $Z$ elements.

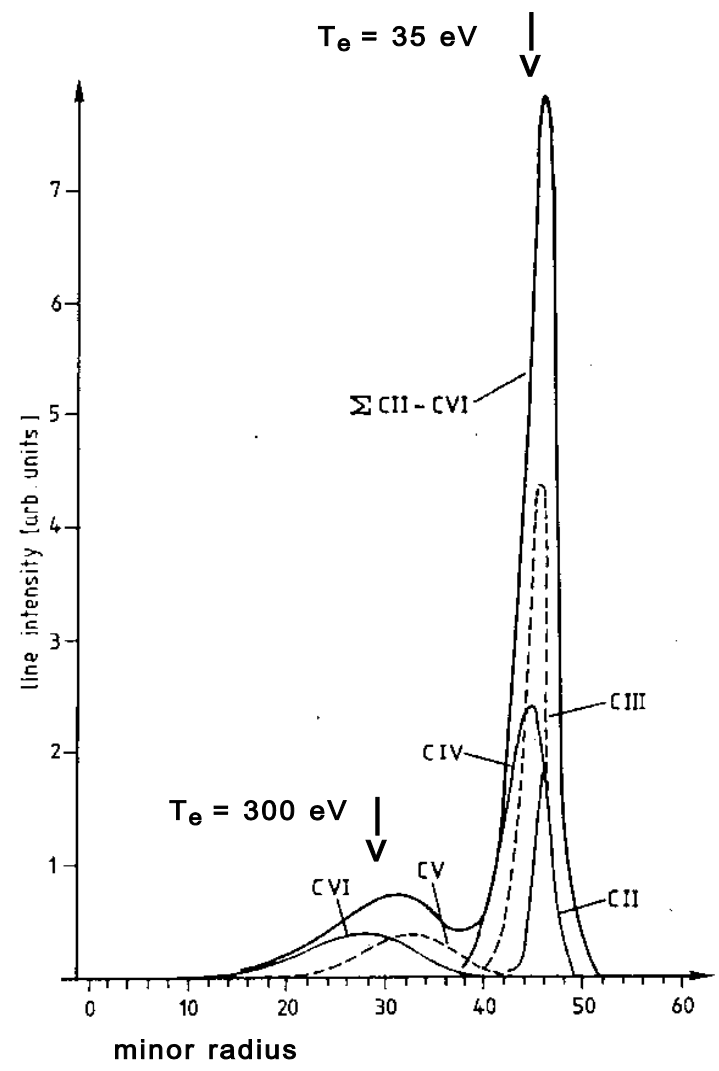

Figure 3 Radial distribution of the radiated power from carbon ions calculated with a 1d-transport code for TEXTOR conditions

In TEXTOR experiments $\mathrm{E}_{\mathrm{rad}}$ has been determined from measurements of impurity fluxes $\Gamma_{\mathrm{i}}$ and $\mathrm{P}_{\mathrm{rad}}{ }^{8}$ and has been calculated with a 1-d transport code ${ }^{9}$. Results for the impurities C, $\mathrm{O}$ and Ne are shown in Fig. 4, where $\mathrm{E}_{\mathrm{rad}}$ is plotted versus the $\mathrm{T}_{\mathrm{e}}$ values at which the particles enter the plasma. When comparing $\mathrm{E}_{\mathrm{rad}}$ and $\mathrm{P}\left(\mathrm{T}_{\mathrm{e}}\right)$ we have to keep in mind that in a 0 -d calculation (e.g. Fig. 1) all the radiation is emitted at a specific $T_{e}$ value, whereas the $T_{e}$ values shown for the 1-d case (Fig. 4) represent the starting points of radial profiles.

\section{I.B. $T_{e}$ - dependence and extent of the radiating boundary}

The characteristic dependence of radiation per particle on $T_{e}$ is already evident from the behaviour of a single transition as shown in Fig. 1, where the ratio of rate coefficients exhibits a pronounced increase with decreasing $\mathrm{T}_{\mathrm{e}}$. This is typical for all transitions and charge states. Thus, after summation also $\mathrm{E}_{\text {rad }}$ as well as $\mathrm{P}\left(\mathrm{T}_{\mathrm{e}}\right)$ will have a similar $\mathrm{T}_{\mathrm{e}}$-dependence as can be seen in Figs. 2 and 4. Such a behaviour is favorable for generating a cold radiating boundary, since with a decrease of $T_{e}$ the radiation level is rising without employing more impurities. On the other hand, this property can lead to instabilities as will be discussed later.

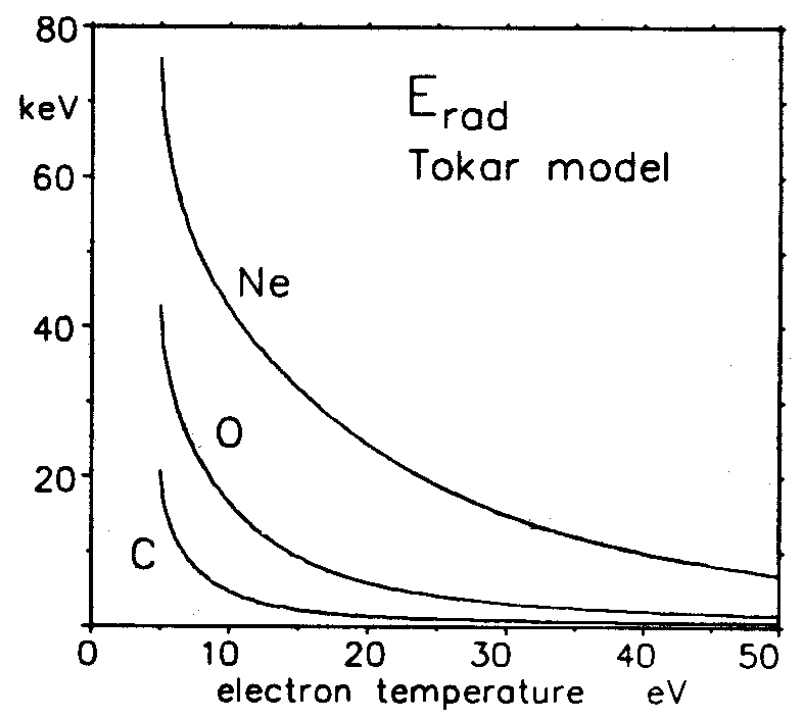

Figure 4 The radiation potential as a function of the edge electron temperature for TEXTOR conditions

The number of excitations per particle can also be illustrated with the following formulas for the Born-Coulomb rate coefficients for ionization and excitation ${ }^{3}$

$$
\begin{aligned}
& <\sigma_{\mathrm{i}} \mathrm{V}>=10^{-8}\left|\frac{\mathrm{R}_{\mathrm{y}}}{\mathrm{E}_{\mathrm{o}}}\right|^{3 / 2} \exp \left(-\frac{\mathrm{E}_{\mathrm{o}}}{\mathrm{T}_{\mathrm{e}}}\right) \mathrm{Q}_{\mathrm{i}} \frac{\mathrm{G}_{\mathrm{i}}\left(\mathrm{E}_{\mathrm{o}} / \mathrm{T}_{\mathrm{e}}\right)}{21+1} \\
& <\sigma_{\mathrm{e}} \mathrm{V} \geq 10^{-8}\left|\frac{\mathrm{R}_{\mathrm{y}}}{\mathrm{E}_{\mathrm{o}}-\mathrm{E}_{1}}\right|^{3 / 2}\left(\frac{\mathrm{E}_{1}}{\mathrm{E}_{\mathrm{o}}}\right)^{3 / 2} \exp \left(-\frac{\Delta \mathrm{E}_{\mathrm{o}}}{\mathrm{T}_{\mathrm{e}}}\right) \mathrm{Q}_{\mathrm{k}} \frac{\mathrm{G}\left(\Delta \mathrm{E}_{\mathrm{o}} / \mathrm{T}_{\mathrm{e}}\right)}{21+1}
\end{aligned}
$$

where the following parameters are used : the Rydberg constant $\mathrm{R}_{\mathrm{y}}$, the ionization energy from the ground state $E_{0}$, the ionization energy from the excited state $\mathrm{E}_{1}$ and $\Delta \mathrm{E}=\mathrm{E}_{\mathrm{o}}-\mathrm{E}_{1}$. $\mathrm{Q}_{\mathrm{i}}$ is a parameter depending on the quantum numbers of the electron shell. If we neglect the weak variation of the factors $G$ and $G_{i}$ for a rough estimate and multiply the number of excitations per ion by the average excitation energy $\Delta \mathrm{E}$ then we obtain the following relation for the power radiated from this level

$$
\Delta \mathrm{E} \frac{<\sigma_{\mathrm{e}} \mathrm{V}>}{<\sigma_{\mathrm{i}} \mathrm{V}>} \propto \exp \left(\mathrm{E}_{1} / \mathrm{T}_{\mathrm{e}}\right) \frac{\mathrm{E}_{1}^{3 / 2}}{\Delta \mathrm{E}^{1 / 2}}
$$


which clearly exhibits the strong drop of radiation with increasing $\mathrm{T}_{\mathrm{e}}$.

In the case of an edge plasma, where the plasma parameters vary strongly in radial direction, the location of radiation is essentially determined by the actual $\mathrm{T}_{\mathrm{e}}$-profile. According to the results obtained from a 1-d transport code shown in Fig. 3 for the case of carbon the different ionization states contribute in a distinct way. The Li- and Be-like states (C III, C IV) contribute more (factor of 2-3) to the total radiation than the He- or H-like ions (C V, C VI). This is due to the fact that the relative difference between ionization and excitation energies is significantly larger in the case of the Liand Be-like ions leading to more excitations per ionization. The influence of these energy levels is also reflected in equs. 9-11.

\begin{tabular}{|c|c|c|c|c|c|c|c|c|c|}
\hline & & & eleme & & & & & & \\
\hline & $\mathrm{Li}$ & $\mathrm{Be}$ & B & $\mathrm{C}$ & $\mathrm{N}$ & $\mathrm{O}$ & $\mathrm{F}$ & $\mathrm{Ne}$ & $\mathrm{Si}$ \\
\hline $\begin{array}{l}\text { electr. } \\
\text { config. } \\
\text { like }\end{array}$ & 3 & 4 & 5 & 6 & 7 & 8 & 9 & 10 & 14 \\
\hline $\mathrm{H}$ & 122 & 218 & 340 & 490 & 667 & 871 & 1103 & 1360 & 2673 \\
\hline $\mathrm{He}$ & 75 & 153 & 259 & 392 & 552 & 739 & 953 & 1196 & 2437 \\
\hline $\mathrm{Li}$ & 5 & 18 & 38 & 65 & 98 & 138 & 185 & 239 & 523 \\
\hline $\mathrm{Be}$ & & 9 & 25 & 48 & 76 & 114 & 157 & 208 & 476 \\
\hline B & & & 8 & 24 & 48 & 77 & 114 & 158 & 401 \\
\hline C & & & & 11 & 30 & 55 & 87 & 126 & 351 \\
\hline $\mathrm{N}$ & & & & & 14 & 35 & 63 & 92 & 303 \\
\hline $\mathrm{O}$ & & & & & & 14 & 35 & 63 & 246 \\
\hline $\mathrm{F}$ & & & & & & & 17 & 41 & 205 \\
\hline $\mathrm{Ne}$ & & & & & & & & 22 & 166 \\
\hline $\mathrm{Na}$ & & & & & & & & & 54 \\
\hline $\mathrm{Mg}$ & & & & & & & & & 33 \\
\hline $\mathrm{Al}$ & & & & & & & & & 16 \\
\hline $\mathrm{Si}$ & & & & & & & & & 8 \\
\hline $\begin{array}{c}\Sigma \mathrm{E}_{\mathrm{i}} \\
\text { all }\end{array}$ & 202 & 398 & 670 & 1030 & 1485 & 2043 & 2714 & 3505 & 7893 \\
\hline $\begin{array}{c}\Sigma \mathrm{E}_{\mathrm{i}} \\
-\mathrm{Li}\end{array}$ & 5 & 27 & 71 & 148 & 267 & 433 & 658 & 949 & 2783 \\
\hline
\end{tabular}

Table 1 Ionization energies in $\mathrm{eV}$ and their sum over all levels $\left(\Sigma \mathrm{E}_{\mathrm{i}}\right.$ all $)$ and excluding the $H$ - and He-like states $\left(\Sigma \mathrm{E}_{\mathrm{i}}-\mathrm{Li}\right)$

Most important is the fact that the CVI and CV radiation is distributed over a broader radial range than the radiation from the lower ionization states as is evident from Fig. 3. As a consequence the peak radiation densities differ by about a factor of 10 . This is beneficial for the purpose of generating a radiating plasma boundary. The larger spread of radiation for the $\mathrm{H}$ - and He-like ions is caused by their relative high ionization energies $\mathrm{E}_{\mathrm{o}}$ leading to an enhancement of their ionization length (equ.2). The jump of $E_{o}$ when going from the Li-like to the He-like state is typical for all elements as can be seen in table 1 .

Based on these properties we may conclude that the extent of the radiating layer towards higher $T_{e}$ is limited essentially by the ionization of the Li-like ions. A crude estimate about the $T_{e}$ values at which a particle is ionized can be obtained by calculating the ionization length $\lambda_{\mathrm{i}}$ according to equ. 2 . The strong $\mathrm{T}_{\mathrm{e}}$-dependence of $\left\langle\sigma_{\mathrm{i}} \mathrm{v}\right\rangle$ will cause a drastic drop of $\lambda_{\mathrm{i}}$ with increasing temperature. We may define the location at which $\lambda_{\mathrm{i}}$ drops below a certain value as the radius of ionization (and radiation). Experiments as well as model calculations show that the ionization occurs at an electron temperature corresponding to $1 / 2$ to $1 / 3$ of the ionization energy $E_{o}$ of the particular ion. Therefore a guess at the extent of a radiating layer is given by the location of $\mathrm{T}_{\mathrm{e}}=\mathrm{E}_{\mathrm{o}} / 2$ for the Li-like ion stage. It is interesting to compare the radiated energy per particle $\mathrm{E}_{\mathrm{rad}}$ with the total energy needed for ionization. Comparing table 1 with figure 4 shows that about 10-40 times more energy can be radiated than is necessary for ionization up to the He-like state $\left(\Sigma \mathrm{E}_{\mathrm{i}}(-\mathrm{Li})\right)$.

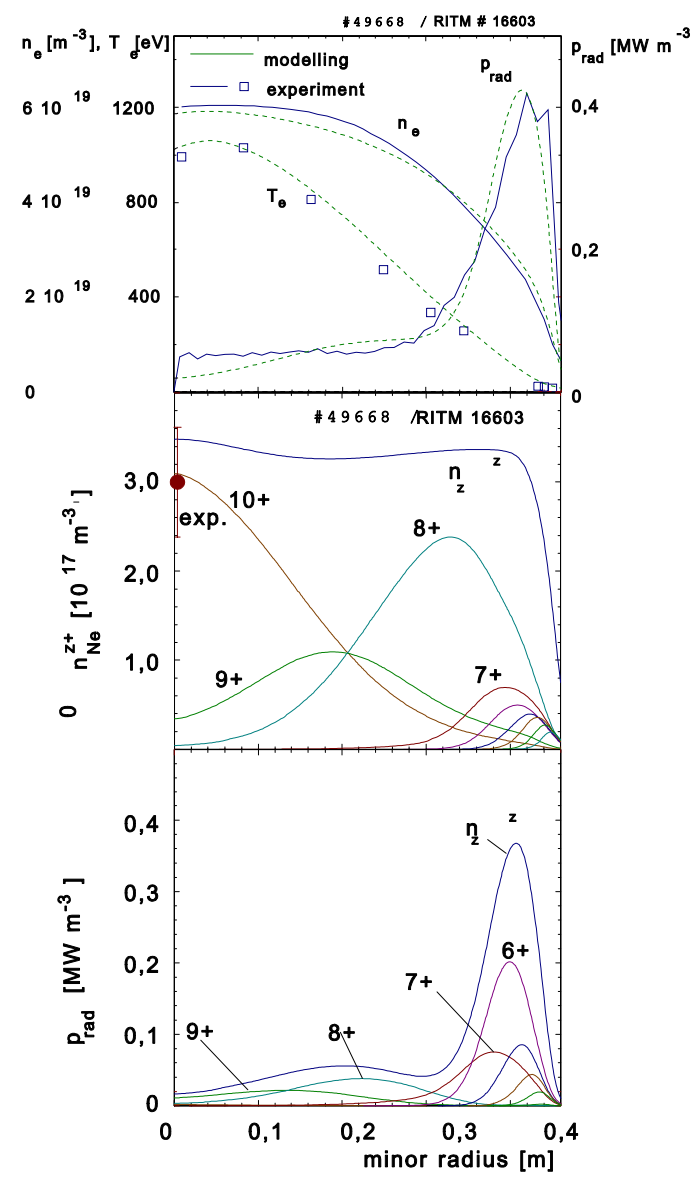

Figure 5 Radiation cooling with neon; profiles of $n_{e}, T_{e}$ and radiated power measured in TEXTOR and model calculations with the code RITM showing the neon ion densities and the corresponding radiation

For neon the main radiation is expected to go up to $\mathrm{T}_{\mathrm{e}} \approx 120 \mathrm{eV}$. This can be confirmed by experimental data obtained on TEXTOR shown in Fig. 5. With neon puffing a high level of radiation $\left(\mathrm{P}_{\mathrm{rad}} / \mathrm{P}_{\text {heat }}=85 \%\right.$ in this case $)$ has been obtained. $\mathrm{T}_{\mathrm{e}}$ in the centre 
remains constant (or even increases slightly), whereas $T_{e}$ at the edge is significantly reduced. This proves that selective edge cooling is possible. The drop in $\mathrm{T}_{\mathrm{e}}$ starts at a radius of $\mathrm{r} / \mathrm{a} \approx 0.75$, which coincides with the extent of the main part of the radiative layer and an electron temperature of about $120 \mathrm{eV}$. A strong drop of the radiation occurs when the Li-like ions $\left(\mathrm{Ne}^{7+}\right)$ are ionized, as is clearly seen in Fig. 5. According to this simple rule we may estimate the extent of the radiative layer for all tokamaks only based on the specific $\mathrm{T}_{\mathrm{e}}$-profile. This is the main effect. Variation of transport will be a second order effect. An important consequence is that in future devices like ITER, where much higher $T_{e}$ values will develop, the radiative layer will be confined to a much smaller fraction of the whole volume.

\section{I.C. Radiation in a divertor configuration}

The strong plasma flow inside the divertor towards the divertor plates changes the radiation properties of the edge plasma significantly ${ }^{10,11,12}$. Neutral particles recycling at the target plate are ionized inside the divertor leading to rather high electron densities in the divertor chamber. The impurities released from the target plates, which are also mostly ionized inside the divertor, are partly flushed back due to friction forces caused by the strong flow of the background plasma, in particular in regions close to the target plate, or are pushed upstream due to thermal forces caused by the temperature gradient along the magnetic field lines. Thus, on the one hand we get radiation from impurities inside the divertor and on the other hand impurities reaching the main chamber can generate a radiating belt at the edge plasma in a similar way as for a limiter configuration. Although the radiation density inside the divertor is generally much higher than that of the radiating belt in the main chamber, the total radiated power can be about the same because of the much larger volume in the main chamber.

\section{EXPERIMENTAL RESULTS AND LIMITATIONS OF THE CONCEPT}

\section{III.A. The radiation level}

On TEXTOR it has been shown first that already with light species, like $\mathrm{Ne}$ or $\mathrm{Si}$, which provide selective edge cooling, a significant fraction of the total power can be radiated ${ }^{13,14}$. These light impurities may serve for the realization of the concept of the cold radiating plasma boundary. The discharges are stable and quasistationary. In fig. 6 some data from a series of shots is shown with various amounts of neon at a medium line-averaged electron density $\left(\mathrm{n}_{\mathrm{e}}=4.10^{13} \mathrm{~cm}^{-3}\right)$, a medium heating power level $(1.6 \mathrm{MW})$ and a flat top time for the radiation of about $1 \mathrm{~s}$. The radiation level can rise close to $100 \%$. Similar results have been obtained at a high power level, where a radial power flux density of $0.11 \mathrm{MW} / \mathrm{m}^{2}$ to the edge is obtained. In this case the convective transport entering the scrape-off layer can be reduced by radiation to $0.02 \mathrm{MW} / \mathrm{m}^{2}$. In TEXTOR it has been demonstrated that such conditions can be kept quasi-stationary ${ }^{15}$ (see also fig.8).

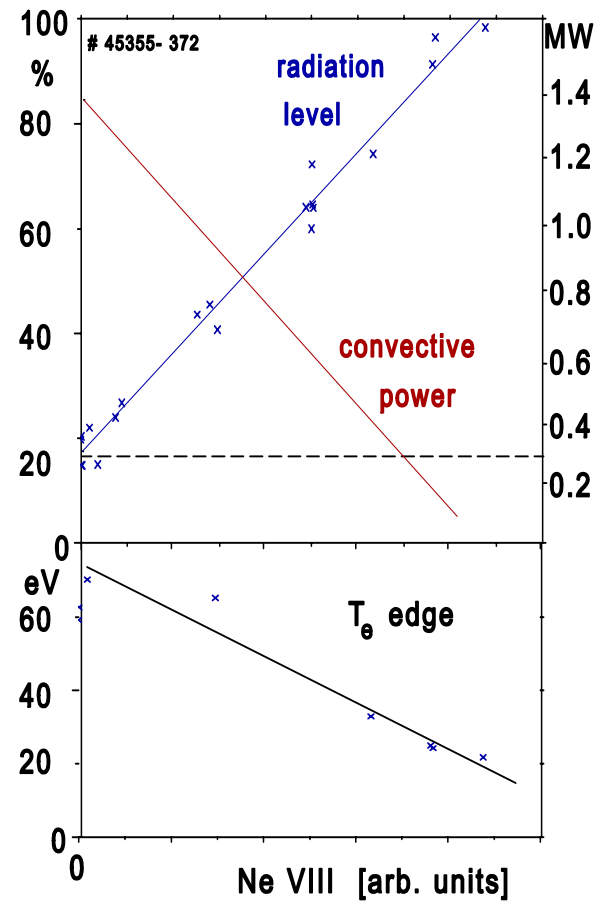

20

Figure 6 Radiation level, convective power to limiter and edge $T_{e}$ as a function of the amount of injected neon

Also in tokamaks with a divertor a high fraction of the heating power can be removed by radiation from the plasma boundary. This has been demonstrated first on ASDEX-Upgrade with the help of neon injection ${ }^{16,17}$. An essential element of both experiments - TEXTOR and ASDEX-U - is the use of a feedback system for neon as a key element for achieving quasistationary conditions. Meanwhile radiation cooling experiments have been performed on many tokamaks by injecting $\mathrm{Ne}, \mathrm{Ar}$ and $\mathrm{N}$. The critical issues in these experiments with a radiative plasma boundary as feed-back control, thermal stability, energy confinement and impurity contamination are discussed in the following.

III.B. Special properties, limitations and critical issues of the concept

Feed-back control of the impurity level

Quasi-stationary operation of discharges at a radiation level close to $100 \%$ is only possible with a very well controlled impurity level. At such a high radiation level already a tiny increase of the impurity concentration can lead to a major disruption. It turns out that a feedback system to control the impurity level is indispensable. In case of neon this has been realized with the help of a gas-puff system and by employing as a sink either a pump limiter (ALT-II in TEXTOR) or a pumped divertor (ASDEX-U). For the control variable we may use a NeVIII line (TEXTOR), which represents the total radiation from neon sufficiently well, or directly a fast bolometer signal giving the total radiation (ASDEX-U, JET). 
The control of silicon is more difficult. In a siliconized machine the intrinsic source for $\mathrm{Si}$ is sputtering, a process which depends strongly on the plasma edge temperature. Generally, with increasing electron density the silicon radiation decreases due to a drop of the sputtering yield. Additional radiation from Si can be obtained e.g. by $\mathrm{Si}_{2} \mathrm{H}_{6}$ puffing, but the possible variation is limited due to the unavoidable density increase arising from the injection of molecules containing hydrogen. The maximum radiation level obtained so far was about $\gamma=75 \%$ with full di-silane fuelling ${ }^{18}$.

\section{Thermal stability}

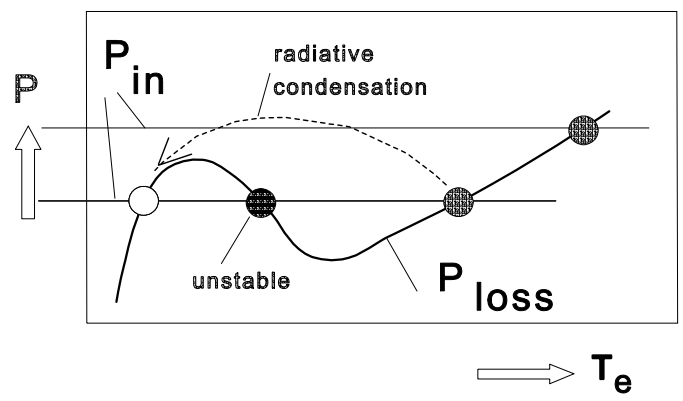

Figure 7 Stable and unstable regimes in the local heat flux balance of a radiating edge plasma

Fig. 7 shows schematically a typical $\mathrm{T}_{\mathrm{e}}$-dependence of the local energy losses $\mathrm{P}_{\text {loss }}$ in an edge plasma. In steady state the local power input (heating, conduction, convection) has to balance the losses. At high electron temperatures the losses are dominated by charge exchange and ionization processes and therefore increase with $T_{e}$, whereas in a cold plasma the radiative losses dominate showing an increase with decreasing $T_{e}$ (compare with Fig. 4). At the low end of the $T_{e}$ range the losses decrease again because the processes leading to radiation diminish (e.g. particle sources). At high relative input power the solution is in a range where $\delta \mathrm{P}_{\text {loss }} / \delta \mathrm{T}_{\mathrm{e}}>0$, i.e. the system is stable. When lowering the power level (equivalent is increasing the radiation level) a second stable solution at low $\mathrm{T}_{\mathrm{e}}$ may be possible, as is indicated in Fig. 7. An abrupt transition to this state is often observed. On a time scale of 10th of ms zones of cold plasma develop. In low and medium power discharges this can occur poloidally symmetric. Then the whole hot plasma column appears to shrink radially, which is called detachment because the plasma flow to the limiters is nearly vanishing ${ }^{19}$. At high power levels, where normally strong in/out poloidal asymmetries in the heat transport are present, we observe a different phenomenon. Instead of a full detachment a toroidal belt of a cold, high density plasma develops at the location of lowest radial heat flux, that is at the high field side of the tokamak. This highly radiating belt is called a "MARFE" (Multifaceted Asymmetric Radiation From the Edge $)^{20}$. In such a MARFE the electron density is much higher and the electron temperature much lower $\left(\mathrm{n}_{\mathrm{e}}>10^{14} \mathrm{~cm}^{-3} \mathrm{~T}_{\mathrm{e}}<5 \mathrm{eV}\right)$ than in neighbouring plasma regions ${ }^{21}$. The total power radiated from a MARFE is in the order of $20 \%-50 \%$ of the total input power. It is important to note that this radiation may lead in the vicinity of the MARFE to significant peak loads on the wall. Experimentally,
MARFEs can be avoided by operating not too close to the density limit.

The radiation inside the divertor can lead to the phenomenon of divertor detachment. In contrast to the detachment with edge radiation, where the cold plasma layer moves in radial direction (radial temperature gradient), the peak radiation inside the divertor moves parallel to the field lines (parallel temperature gradient) away from the target plate. As a consequence the plasma flow to the plates diminishes. At the x-point normally the heat flux density is high enough to stop this development resulting in a strongly radiating zone located around the x-point which is called x-point $\mathrm{MARFE}^{22}$. It is important to avoid too strong a detachment, because the divertor function would be lost in case of an extremely cold divertor plasma where the ionization front moves too far away from the target.

\section{Energy confinement}

The concept of a cold radiative plasma boundary can only be applied successfully to a fusion reactor if it is compatible with the important requirements on confinement. The energy confinement must be kept sufficiently high and the particle confinement, in particular the confinement of $\mathrm{He}$ ash, must be low enough to allow for a stationary burning plasma.

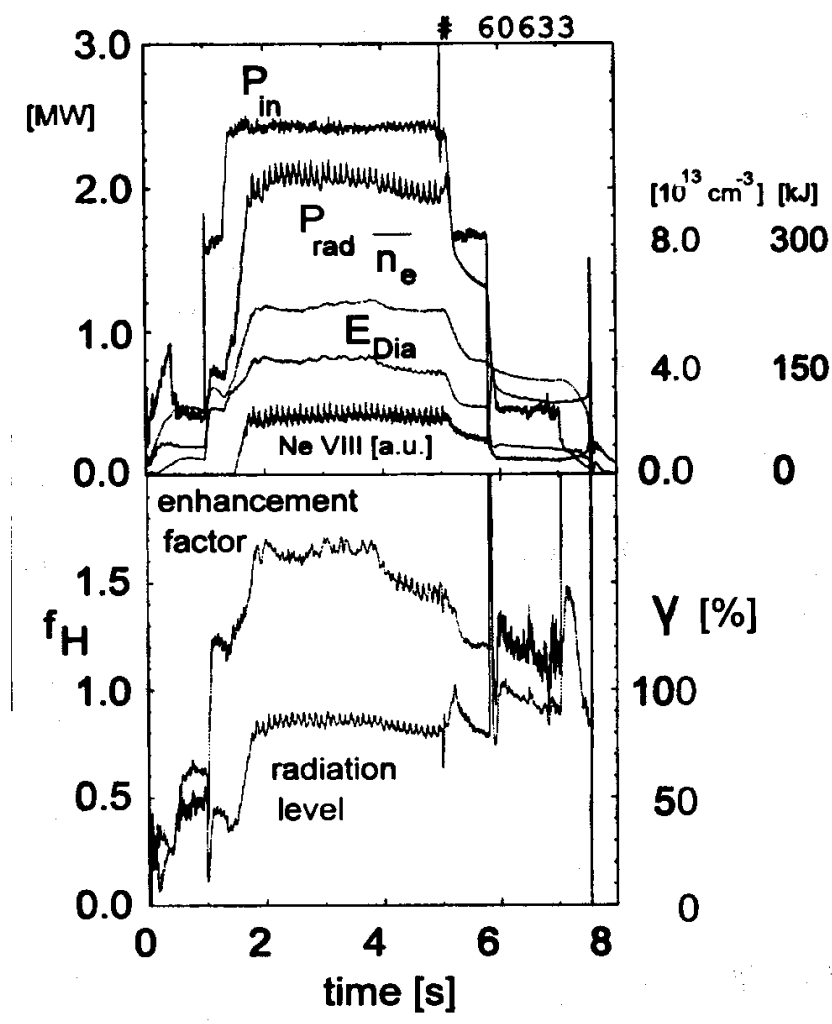

Figure 8 Improved confinement in a long pulse discharge with edge cooling by neon

It is remarkable that under special circumstances strong radiative edge cooling can increase the global energy confinement time. This 
phenomenon has already been observed in earlier experiments ${ }^{23}$, but on a high power level and in conjunction with controlled edge cooling it has first been demonstrated on TEXTOR and ASDEX-U. In fig. 8 a TEXTOR discharge is shown where a clear transition to increased energy confinement is observed with neon injection (displayed by the time trace of the Ne-VIII line radiation). About $80 \%$ of the total input power is radiated. The quality of the energy confinement corresponds to ELM-free H-mode confinement in divertor tokamaks. In TEXTOR this type of discharge is called Radiative Improved mode ${ }^{24}$. A set of ASDEX-U discharges with different radiation levels is shown in fig.9. It is demonstrated that under certain conditions (amount of neon) the energy confinement time follows the H-mode scaling (high confinement mode). Since the power onto the divertor plate nearly diminishes this is called the Complete Detached H-mode (CDH-mode) ${ }^{16,17}$. It is remarkable that also under L-conditions without the characteristic H-mode signatures (e.g. edge transport barrier and ELMs) - in a discharge scenario very similar to the RI-mode- a significant improvement of confinement is observed coming close to that of the CDH-mode (circles in fig 9 at high $\mathrm{P}_{\text {rad }} / \mathrm{P}_{\text {loss }}$ ).

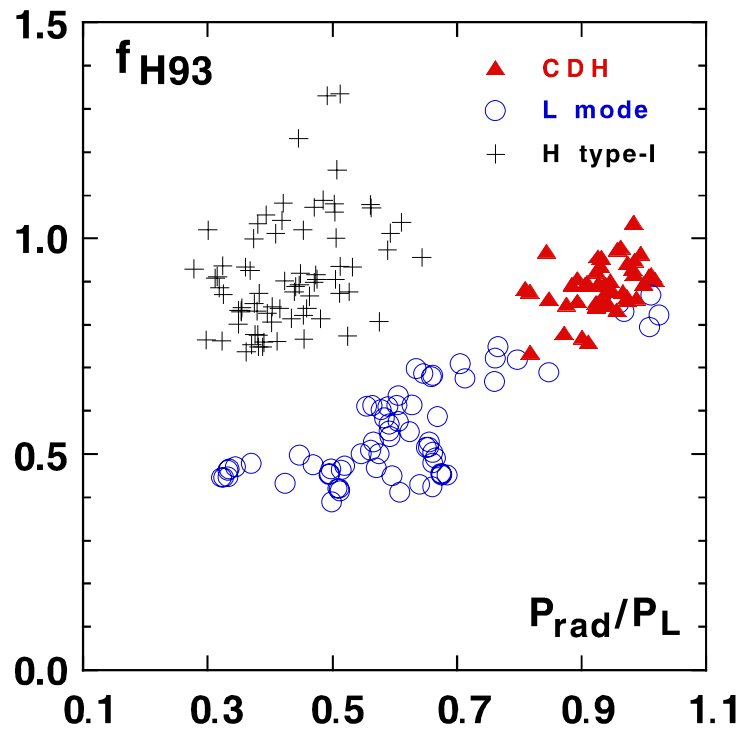

Figure 9 energy confinement normalized to the H-mode scaling for different radiation level with $(C D H)$ and without (L-, H-mode) neon injection in ASDEX-Upgrade

The improvement of energy confinement in radiating mantle plasmas is attributed to the suppression of the so called toroidal ion temperature gradient driven mode as a consequence of the impurity injection $^{25}$. To extrapolate this very attractive confinement regime to reactor scale plasmas experiments in large devices like JET are being conducted with the aim to combine high density operation and good confinement with power exhaust by radiation ${ }^{26}$. In this context the influence of a radiating edge to $\mathrm{H}$-mode properties is a crucial issue. It has been observed that above a certain radiation level the edge plasma pressure gradients can be reduced such that the H-mode properties are lost with the consequence of reduced confinement. It is an issue of ongoing research to optimize the combination of high confinement and the feed-back injection of radiating impurities.

$\underline{\text { Radial transport and He-exhaust }}$

We can identify two aspects in which impurity transport is a critical issue with respect to radiation cooling : a) efficient He removal (ash exhaust) has to be possible with a "cold" plasma edge and b) the build up of impurity concentrations in the centre - helium as well as other impurities including those used for radiation cooling must stay below a critical level.

For efficient He-removal extreme divertor detachment has to be avoided, because the pumping efficiency might become to small. A much more critical issue is the amount of impurities being transport and accumulated in the plasma centre. From the conditions for a burning fusion plasma follows an upper limit for the impurity concentration in the plasma centre ${ }^{27}$. The radial transport of helium and its exhaust properties determine the margin left for other impurities than helium acceptable in the plasma core. Taking into account also the impurities coming from erosion processes a certain margin will be left for those special impurities needed to enhance and control the radiation cooling. From this follows also a maximum level of radiation. Thus the concept of a radiating boundary is only realistic under conditions with sufficient Heexhaust and well controlled impurity contamination from wall erosion.

\section{CONCLUSIONS}

Edge radiation can significantly help to solve the power exhaust problem: radiation distributes the energy on large areas of the wall, thus avoiding or reducing the peak power loads which appear at the strike point of the divertor target. The concept of a radiating plasma mantle is now also part of the plasma scenarios foreseen for ITER. In ITER a significant level of radiation is foreseen (at least $60 \%$ of the effective heating power) to bring the peak load to the wetted area down to values below $10 \mathrm{MW} / \mathrm{m}^{2}$. With a maximum of radiation $(>90 \%)$ the average heat load to the main vessel wall would not exceed $0.15 \mathrm{MW} / \mathrm{m}^{2}$.

With this method the long standing problem of excess average heat loads seems to be solved, provided all the other requirements for a burning fusion plasma are also fulfilled:

- $\quad$ acceptable fuel dilution by impurities in the plasma centre

- $\quad$ sufficient energy confinement

- $\quad$ no extreme divertor detached

The optimization of this concept based on improved understanding of the underlying mechanisms is a key issue of ongoing research in present machines and later in particular on ITER.

\section{References}

1. D.J. Campbell Physics of Plasmas 85 (2001) 2041

2. K.L. BELL et al., "Atomic and molecular data for Fusion," UKAEA-Report CLM-R216 (1982) 
3. I.I. SOBELMAN et al., "Excitation of Atoms and Broadening of Spectral Lines," Springer Verlag Berlin (1981)

4. R.V. JENSEN AND D.E. POST, Atomic Data and Nuclear Data Tables Vol. 20, No. 5 (1977)

5. SUMMERS H.P. AND MCWHIRTER R.W.P. in "Applied Atomic Collision Physics", Vol.2, eds. Massey et al. Academic Press London (1984)

6. J. NEUHAUSER et al., Nucl. Fus. 2612 (1986)

7. H.A. CLAASSEN et al. J. Nucl. Mater. 176-177 (1990) 398

8. U. SAMM et al., "Radiation cooling with intrinsic and injected impurities in the plasma boundary of a limiter tokamak," in Proc. of the 18th Europ. Conf. on Plasma Physics and Controlled Fusion Berlin (1991) Vol.3, 157

9. M.Z. TOKAR', Plasma Phys. Contr. Fusion 36 (1995) 1819

10. M.Z. TOKAR', Transactions of Fusion Technology 37 (2000)

11. B. UNTERBERG and U.SAMM, these lectures

12. P.C.STANGEBY, The Plasma Boundary of Magnetic Fusion Devices, Plasma Physics Series, IOP Publishing Ltd, Bristol UK (2000)

13. U. SAMM et al., "Plasma edge cooling by impurity radiation in a tokamak," Proc. of the 14th Inter. Conf. on Plasma Physics and Controlled Nucl. Fusion IAEA, Würzburg (1992)

14. U. SAMM et al., Plasma Phys. Contr. Fusion B167-B175 (1993)

15. A.M. MESSIAEN et al. Phys. Rev. Lett. 77 (1996) 2487

16. A. KALLENBACH et al., Nucl. Fusion 3510 (1995) 1231

17. J. NEUHAUSER et al. Plasma Phys. Contr. Fusion 37 (1995) A 37

18. U. SAMM et al., "Plasma Edge Physics with Siliconization in TEXTOR", Journal of Nucl. Mater. 220-222 (1995) 25

19. U. SAMM et al., "Properties of detached plasmas", $K F A$ Report Jül-2123 (1987)

20. B. LIPSCHULTZ, "Review of MARFE-phenomena in tokamaks," J. Nucl. Mater. 145-147 (1989) 15

21. G. SERGIENKO et al., "Investigation of MARFEs in TEXTOR," in Proc. of the 20th Europ. Conf. on Plasma Physics and Controlled Fusion Lisbon (1993) II 667

22. S. KONOSHIMA et al., J. Nucl. Mater. 313-316 (2003) 888

23. E.A. LAZARUS et al., "Confinement in Beam-Heated Plasmas: The Effect of Low-Z Impurities", in Nucl. Fusion 25 2 (1985) 135

24. R.R. Weynants et al Nucl. Fusion 39 (1999) 1637

25. M.Z. TOKAR', Phys. Rev. Lett. 84 (1995) 895

26. J. ONGENA et al., Physics of Plasmas, 8 (5) 2188. (2001)

27. D. REITER et al., "Helium removal from tokamaks," Plasma Physics and Controlled Fusion Vol.33, No. 13 (1991) 1579 